\title{
An Aquaporin-2 Water Channel Mutant Which Causes Autosomal Dominant Nephrogenic Diabetes Insipidus Is Retained in the Golgi Complex
}

\author{
Sabine M. Mulders, ${ }^{\star}$ Daniel G. Bichet, ${ }^{\ddagger}$ Johan P.L. Rijss, ${ }^{\star}$ Erik-Jan Kamsteeg, ${ }^{\star}$ Marie-Françoise Arthus, ${ }^{\ddagger}$ Michele Lonergan, ${ }^{\ddagger}$ \\ Mary Fujiwara, ${ }^{\S}$ Kenneth Morgan, " Richtje Leijendekker, ${ }^{\rrbracket}$ Peter van der Sluijs, $"$ Carel H. van Os, ${ }^{\star}$ and Peter M.T. Deen ${ }^{\star}$ \\ *Department Of Cell Physiology, University of Nijmegen, 6500 HB Nijmegen, The Netherlands; ${ }^{\ddagger}$ Unité de Recherche Clinique, Centre de \\ Recherche et Service de Nephrologie, Hôpital du Sacré-Coeur, Department de Médicine, Université de Montréal, Montréal, Québec,

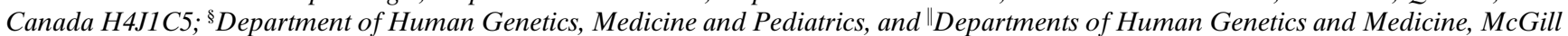 \\ University, Montreal, Quebec, H3A 1A1 Canada; and "Department of Cell Biology, Utrecht University School of Medicine, 3584 CX \\ Utrecht, The Netherlands
}

\begin{abstract}
Mutations in the aquaporin-2 (AQP2) water channel gene cause autosomal recessive nephrogenic diabetes insipidus (NDI). Here we report the first patient with an autosomal dominant form of NDI, which is caused by a G866A transition in the $A Q P 2$ gene of one allele, resulting in a $\mathrm{E} 258 \mathrm{~K}$ substitution in the C-tail of AQP2. To define the molecular cause of NDI in this patient, AQP2-E258K was studied in Xenopus oocytes. In contrast to wild-type AQP2, AQP2E258K conferred a small increase in water permeability, caused by a reduced expression at the plasma membrane. Coexpression of wild-type AQP2 with AQP2-E258K, but not with an AQP2 mutant in recessive NDI (AQP2-R187C), revealed a dominant-negative effect on the water permeability conferred by wild-type AQP2. The physiologically important phosphorylation of $\mathrm{S} 256$ by protein kinase A was not affected by the E258K mutation. Immunoblot and microscopic analyses revealed that AQP2-E258K was, in contrast to AQP2 mutants in recessive NDI, not retarded in the endoplasmic reticulum, but retained in the Golgi compartment. Since AQPs are thought to tetramerize, the retention of AQP2-E258K together with wild-type AQP2 in mixed tetramers in the Golgi compartment is a likely explanation for the dominant inheritance of NDI in this patient. (J. Clin. Invest. 1998. 102:57-66.) Key words: mutation - aquaporin-2 • oocytes • routing • Golgi apparatus
\end{abstract}

\section{Introduction}

Water transport in the kidney occurs through specialized channels called aquaporins. They play an important role in reabsorption of water and in concentration of urine. At least four different aquaporins are thought to participate in these processes (AQP1-4) ${ }^{1}(1-6)$, but so far, only one water channel, AQP2, has been shown to be essential $(7,8)$. AQP2 is uniquely

Address correspondence to Peter M.T. Deen, Ph.D., 162, Dept. of Cell Physiology, University of Nijmegen, P.O. Box 9101, 6500 HB Nijmegen, The Netherlands. Phone: 31-243617347; FAX: 31-243540525; E-mail: peterd@sci.kun.nl

Received for publication 21 December 1997 and accepted in revised form 21 April 1998.

J. Clin. Invest.

(C) The American Society for Clinical Investigation, Inc. 0021-9738/98/07/0057/10 \$2.00

Volume 102, Number 1, July 1998, 57-66

http://www.jci.org localized to subapical storage vesicles in principal cells of the collecting duct $(9,10)$. Upon binding of arginine vasopressin (AVP) to the V2 receptor (V2R) at the basolateral side of the cell, intracellular cAMP levels increase, resulting in phosphorylation of AQP2 and other proteins by protein kinase A (PKA). Subsequently, transport vesicles containing the AQP2 water channels translocate to and fuse with the apical plasma membrane. Upon removal of AVP, AQP2 is internalized by endocytosis (9-12).

Nephrogenic diabetes insipidus (NDI) is a disease which is characterized by the inability of the kidney to concentrate urine in response to AVP. To date, the molecular cause of two patterns of inheritance for this disease has been described. The $\mathrm{X}$-linked form of NDI has been shown to be caused by mutations in the gene coding for the $\mathrm{V} 2$ receptor $(13,14)$. Mutations in the $A Q P 2$ gene have been shown to be the cause of the autosomal recessive form of NDI $(7,8)$. In Xenopus oocytes, mutant AQP2 proteins as encoded in this form of NDI were shown to be retarded in the endoplasmic reticulum (ER) and therefore impaired in their routing to the plasma membrane $(8,15,16)$.

Here, we report the first family in which NDI segregates in an autosomal dominant trait. To understand the molecular cause of NDI in the patients of this family, we identified the mutation in their $A Q P 2$ genes, and analyzed the expression, routing, and function of the encoded mutant AQP2 protein in Xenopus oocytes.

\section{Methods}

Patients. The proband (436) is a 37-yr-old female patient with congenital NDI and a 24-h urinary output of 10-12 liters $(164 \mathrm{ml} / \mathrm{kg}$ of body weight $/ 24 \mathrm{~h}$ ). She had a documented lifelong history of polyuria and polydipsia and normal or elevated plasma concentrations of AVP. Her daughter (724), born in 1985, progressively showed increased polyuric-polydipsic symptoms and her 24-h urinary output was measured recently at 6 liters $(136 \mathrm{ml} / \mathrm{kg}$ of body weight $/ 24 \mathrm{~h})$. dDAVP infusion studies were carried out as described previously $(0.3$ $\mu \mathrm{g} / \mathrm{kg}$ of body weight infused in $20 \mathrm{~min}$ ) (17).

Genetic analysis. The $V 2 R$ gene of the proband and her daughter was sequenced with previously described methods $(18,19)$. The $A Q P 2$ gene of all the family members was amplified using a forward primer $5^{\prime}$ to the coding region of exon 1 ( $5^{\prime}$-GCGAGAGCGAGTGCCCG-3') and a reverse primer $3^{\prime}$ to the coding region of exon 4 except for the terminal three codons (5'-GCGGCCCTCAGG-

1. Abbreviations used in this paper: $\mathrm{AQP} 2$, aquaporin-2 water channel; AVP, arginine vasopressin; ER, endoplasmic reticulum; HbA, homogenization buffer A; NDI, nephrogenic diabetes insipidus; $\mathrm{P}_{\mathrm{f}}$, water permeability; PKA, protein kinase A; V2R, vasopressin type 2 receptor; wt, wild-type. 
CCT- $\left.3^{\prime}\right)$. With the generated $A Q P 2$ gene PCR fragment as a template, the coding regions of exons 1-4 were amplified using flanking primers and were manually sequenced as described (18). A G866A transition was identified, resulting in a substitution of a lysine for a glutamic acid (E258K), which correlated perfectly with the polyuric phenotype. Haplotype analysis was carried out using $A Q P 2$ flanking markers (20).

DNA constructs and transcription. To introduce the E258K mutation in an AQP2 expression construct (pT7Ts $A Q P 2)$ (7), the G866A transition was introduced in the human $A Q P 2$ cDNA using the Altered Sites II in vitro mutagenesis kit (Promega, Madison, WI), with the following forward primer: 5'-CGGCAGTCGGTGAAGCTTCACTCGCCGCAG-3'. Besides the G866A transition, this primer introduces a $\mathrm{G}$ to $\mathrm{T}$ transition at position 871. In this way, a HindIII restriction site is created close to the mutation, without changing the amino acid sequence. A NarI-KpnI fragment of $174 \mathrm{bp}$, containing the transitions, was isolated by gel electrophoresis and inserted into the corresponding sites of pT7Ts $A Q P 2$. Using the same technique, an $A Q P 2-S 256 \mathrm{~A}$ mutant was made by introduction of a T860G transition in the $A Q P 2$ cDNA (forward primer 5'-GACGGCGGCAGGCGGTGGAGCTGC-3'). Next, a 282-bp BamHI-KpnI fragment was isolated by gel electrophoresis and inserted into the corresponding sites of pT7Ts $A Q P 2$. A truncated $A Q P 2, \mathrm{AQP} 2-\mathrm{R} 253^{*}$, was created by digestion of pT7Ts $A Q P 2$ with $\mathrm{BsgI}$ and KpnI. Subsequently, the sites were blunt-ended with T4 DNA polymerase and ligated. In this way, the nucleotides coding for all amino acids of AQP2 following R253 were deleted, except for the final amino acids K270 and A271. The AQP2-R187C construct was as described previously (7). Clones that were identical to the wild-type ( $w t)-A Q P 2$ nucleotide sequence except for the transitions or deletions were selected by restriction enzyme analysis and subsequent sequence analysis (21). The obtained pT7Ts $A Q P 2$ constructs were linearized with SalI and capped cRNA transcripts were synthesized in vitro using T7 RNA polymerase according to Promega's (1991) Protocols and Principles guide, except that $1 \mathrm{mM}$ final concentrations of nucleotide triphosphates and 7-methyl-di-guanosine triphosphate were used. The cRNAs were purified and dissolved in DEPC-treated water. The integrity of the cRNAs was checked by agarose gel electrophoresis and their concentrations were determined spectrophotometrically.

Water permeability $\left(P_{f}\right)$ measurements. Oocytes were isolated from Xenopus laevis, and defolliculated by digestion at room temperature for $2 \mathrm{~h}$ with collagenase A $(2 \mathrm{mg} / \mathrm{ml}$; Boehringer Mannheim, Mannheim, Germany). Stage 5 and 6 oocytes were selected and incubated at $18^{\circ} \mathrm{C}$ in modified Barth's solution supplemented with gentamycin $(50 \mu \mathrm{g} / \mathrm{ml})$ (7). The day after isolation, oocytes were injected with water or $0.3-10$ ng cRNA, and analyzed after 2 or $3 \mathrm{~d}$ in a swelling assay as described before (7). Oocyte swelling was performed at $22^{\circ} \mathrm{C}$ after transfer from 200 to 20 mosM (for the AQP2-E258K mutant) or 200 to 70 mosM (for other AQP2 proteins). In all experiments, the data are expressed as means \pm SE. Overall statistical significance was determined by ANOVA. In the case of significance $(P<0.05)$, individual groups were compared by contrast analysis according to Bonferroni.

Antibodies. AQP2:257-271 is an affinity-purified antibody raised against the $15 \mathrm{COOH}$-terminal amino acids of rat AQP2 (15). Antibody AQP2:175-269 is directed against the $\mathrm{E}$ loop and COOH-terminal tail of AQP2 and was obtained in the following way: a 282-bp BamHI-KpnI fragment of $A Q P 2$ was cloned in frame behind the cDNA for glutathione $S$-transferase in the expression vector pGEX3X (Pharmacia Biotech, Uppsala, Sweden) and transfected to DH5 $\alpha$ bacteria. Expression from this construct was induced by IPTG and the resulting fusion protein was isolated. Rabbits were primed with $400 \mu \mathrm{g}$ of the fusion protein in Freund's adjuvant. After $3 \mathrm{wk}$, rabbits were boosted with $200 \mu \mathrm{g}$ of fusion protein in incomplete Freund's adjuvant, which was repeated until the titer of the rabbit serum was sufficiently high as tested in an ELISA. Antibodies were affinity-purified by first passing the serum through an Affigel 15 column (Pharmacia Biotech) of a lysate of $\mathrm{DH} 5 \alpha$ bacteria expressing glutathione
$S$-transferase, followed by a column on which the fusion protein was immobilized. Antibodies were eluted with $0.1 \mathrm{M}$ glycine ( $\mathrm{pH} 2.8$ ) and directly neutralized.

${ }^{35}$ S labeling of $A Q P 2$ in Xenopus oocytes. Directly after injection of $10 \mathrm{ng}$ cRNA encoding wt-AQP2, AQP2-E258K, AQP2-S256A, or a coinjection of $5 \mathrm{ng}$ wt-AQP2 and $5 \mathrm{ng}$ AQP2-E258K cRNA, 12 oocytes were incubated in $0.5 \mathrm{ml}$ modified Barth's solution containing $45 \mu \mathrm{Ci}$ TRAN ${ }^{35} \mathrm{~S}$ label (specific activity $1,380 \mathrm{Ci} / \mathrm{mmol}$; ICN Pharmaceuticals, Irvine, CA). The day after injection, the medium was replaced. $2 \mathrm{~d}$ after injection, 10 oocytes were homogenized in $100 \mu \mathrm{l}$ homogenization buffer A (HbA: $20 \mathrm{mM}$ Tris, $\mathrm{pH}$ 7.4, $5 \mathrm{mM} \mathrm{MgCl}_{2}$, $5 \mathrm{mM} \mathrm{NaHPO}_{4}, 1 \mathrm{mM}$ EDTA, $1 \mathrm{mM}$ DTT, $1 \mathrm{mM}$ PMSF, $5 \mu \mathrm{g} / \mathrm{ml}$ leupeptin and pepstatin, and $80 \mathrm{mM}$ sucrose) and oocyte lysates were prepared by centrifugation twice for $10 \mathrm{~min}$ at $125 \mathrm{~g}$ to remove yolk proteins.

Phosphorylation and immunoprecipitation of AQP2. $2 \mathrm{~d}$ after injection, 10 oocytes were homogenized in $100 \mu \mathrm{HbA}$, and 10 oocytes were homogenized in $100 \mu \mathrm{l} \mathrm{HbA}$ without $\mathrm{NaHPO}_{4}$. Of the latter homogenate, proteins were phosphorylated for $30 \mathrm{~min}$ at room temperature in the presence of $5 \mathrm{mU}$ of the catalytic subunit of PKA (Boehringer Mannheim), $5 \times 10^{-5} \mathrm{M}$ forskolin, $10^{-6} \mathrm{M} \mathrm{cAMP}$, and $20 \mu \mathrm{Ci}$ $\left[\gamma^{32} \mathrm{P}\right]$ ATP (Amersham Life Sciences, Buckinghamshire, UK). Of both batches, oocyte lysates were prepared as described above. For immunoprecipitation, $10 \mu \mathrm{l}$ of $10 \%$ SDS was added to the homogenates described above and the homogenate of oocytes labeled with $\left.{ }^{[35} \mathrm{S}\right]$ methionine, and samples were denatured for $30 \mathrm{~min}$ at $37^{\circ} \mathrm{C}$. Next, the lysates were centrifuged at $14,000 \mathrm{rpm}$ for $1 \mathrm{~min}$ at $4^{\circ} \mathrm{C}$, and the supernatant was diluted $10 \times$ with IPP100 $(10 \mathrm{mM}$ Tris, $\mathrm{pH} 8.0$, $100 \mathrm{mM} \mathrm{NaCl}, 0.1 \% \mathrm{NP}-40$, and $0.1 \%$ Tween 20 ). $10 \mu \mathrm{l}$ protein $\mathrm{A}$ agarose beads (Kem-En Tec, Copenhagen, Denmark) was washed three times with IPP500 (10 mM Tris, pH 8.0, $500 \mathrm{mM} \mathrm{NaCl}, 0.1 \%$ $\mathrm{NP}-40$, and $0.1 \%$ Tween 20 ) and rotated $\mathrm{O} / \mathrm{N}$ at $4^{\circ} \mathrm{C}$ in $500 \mu 1 \mathrm{IPP} 500$ containing $3 \mu \mathrm{l}$ of the polyclonal antibody AQP2:257-271 (15). The beads were washed by rotation for $30 \mathrm{~min}$ at $4^{\circ} \mathrm{C}$ with fresh IPP500, followed by three short washes in IPP500 and one wash with IPP100. Lysate samples, equivalent to two oocytes for $\left[{ }^{35} \mathrm{~S}\right]$ methionine- or nonlabeled oocytes, or four oocytes for the ${ }^{32} \mathrm{P}$-labeled oocyte homogenates, were added to the beads and rotated for $2 \mathrm{~h}$ at $4^{\circ} \mathrm{C}$. Subsequently, the beads were washed three times with IPP100. After the last wash, the beads with the labeled proteins were resuspended in sample buffer (2\% SDS, $50 \mathrm{mM}$ Tris, $\mathrm{pH} 6.8,12 \%$ glycerol, $0.01 \%$ Coomassie brilliant blue, and $100 \mathrm{mM}$ DTT). The beads incubated with the unlabeled proteins were resuspended in $100 \mu \mathrm{l} \mathrm{HbA}$ without $\mathrm{NaHPO}_{4}$ and phosphorylated as described above. Next, these beads were also washed three times with IPP100 and resuspended in sample buffer. All samples were denatured for $30 \mathrm{~min}$ at $37^{\circ} \mathrm{C}$, loaded on a $12 \%$ acrylamide gel, and electrophoresed. Gels were stained with Coomassie brilliant blue, processed for autoradiography, and exposed to a film with an intensifying screen at $-80^{\circ} \mathrm{C}$.

Immunoblotting. After addition of the protease inhibitors PMSF (1 $\mathrm{mM})$, leupeptin $(5 \mu \mathrm{g} / \mathrm{ml})$, and pepstatin $(5 \mu \mathrm{g} / \mathrm{ml}), 40-60-\mathrm{ml}$ morning urine samples of the proband, her affected daughter, and her healthy son were spun down at 3,000 $\mathrm{g}$ for $10 \mathrm{~min}$ to remove cellular debris. Subsequently, the urine was concentrated by ultrafiltration using Centricon and microcon devices equipped with YM10 membranes (Amicon Inc., Beverly, MA) according to the protocol provided by the manufacturer and stored at $-20^{\circ} \mathrm{C}$. Creatinine concentrations of unconcentrated urine samples were determined by standard automated techniques. At 2 or $3 \mathrm{~d}$ after injection, homogenates were prepared from eight oocytes in $160 \mu \mathrm{HbA}$ as described above. On the same day, a fraction enriched for plasma membranes was isolated from 25 oocytes according to Wall and Patel (22). Creatinine equivalents of urine samples or homogenates or plasma membrane fractions equivalent to 0.1 oocyte or 8 oocytes, respectively, were denatured for $30 \mathrm{~min}$ at $37^{\circ} \mathrm{C}$ in sample buffer, subjected to $12 \%$ SDS-PAGE electrophoresis (23) and transferred to a nitrocellulose membrane as described (7). Efficiency of protein transfer was checked by staining the membrane with Ponceau red. For immunode- 
tection, the membrane was incubated with a 1:3,000 or 1:10,000 dilution of antibody AQP2:257-271 (15) or antibody AQP2:175-269, followed by an incubation with a 1:5,000 dilution of affinity-purified goat anti-rabbit $\operatorname{IgG}$ conjugated to horseradish peroxidase (Sigma Chemical Co., St. Louis, MO). Proteins were visualized using enhanced chemiluminescence (Boehringer Mannheim). For semiquantification, signals were densitometrically scanned and compared with those from a twofold serial dilution of lysates from AQP2-expressing oocytes.

Fluorescence microscopy and immunogold electron microscopy. $3 \mathrm{~d}$ after injection, remaining vitelline membranes were removed and oocytes were incubated for $1 \mathrm{~h}$ in $1 \% \mathrm{wt} / \mathrm{vol}$ paraformaldehydelysine-periodate fixative (24), dehydrated, and embedded in paraffin. After blocking with $10 \%$ goat serum in TBS, the sections were incubated $\mathrm{O} / \mathrm{N}$ at $4^{\circ} \mathrm{C}$ with the affinity-purified polyclonal antibody AQP2:175-269, diluted 1:500 in 10\% goat serum in TBS. After three washes for $10 \mathrm{~min}$ in TBS, the sections were incubated for $1 \mathrm{~h}$ in a 1:100 dilution of goat anti-rabbit IgG coupled to FITC (Sigma Chemical Co.). The sections were again washed three times for $10 \mathrm{~min}$ in TBS, dehydrated in ethanol, and mounted in mowiol 4-88, containing $2.5 \% \mathrm{NaN}_{3}$. Photographs were taken with a Zeiss Axioskop with epifluorescent illumination with an automatic camera using Kodak EPH P1600X films. For immunogold electronmicroscopy, oocytes were fixed in $2 \%$ paraformaldehyde in $0.1 \mathrm{M} \mathrm{PBS}, \mathrm{pH} 7.4$, and postfixed in $4 \%$ paraformaldehyde. Next, the oocytes were washed in PBS and sliced with a razor blade. Slices were subsequently embedded in $10 \%$ gelatin and stored for cryoultramicrotomy as described (25). Cryosections were prepared as described (26) and labeled with affinity-purified antibody AQP2:257-271 followed by protein A gold.

\section{Results}

Analysis of the NDI family. From the proband (436) and her affected daughter (724) (Fig. 1), whose family history of NDI indicated an autosomal dominant form of inheritance, the V2 receptor gene $(V 2 R)$ was sequenced, since mutations in $V 2 R$ are frequent in contrast to $A Q P 2$ mutations, and since their NDI could be caused by skewed inactivation of the normal $V 2 R$ allele. However, no mutation in the $V 2 R$ gene was found. Subsequently, the $A Q P 2$ gene of all family members was sequenced. In one allele of the $A Q P 2$ gene of the proband, a point mutation in exon 4 was found. This G866A transition in exon 4 leads to a substitution of a lysine for a glutamic acid at position $258(\mathrm{E} 258 \mathrm{~K})$. The same mutation was found in one allele of the $A Q P 2$ gene of the affected daughter. The other members of the family appeared to be homozygous for the normal allele. Haplotype analysis using $A Q P 2$ flanking markers revealed that G866A was a de novo mutation which occurred on the maternal haplotype (mother of the proband; Fig. 1). To determine urinary AQP2 levels, morning urine samples were collected from the proband, her affected daughter, and her healthy son. Immunoblot analysis of creatinine equivalents of these urine samples revealed that unglycosylated AQP2 $(29 \mathrm{kD})$ could be detected in urine from the affected individuals (Fig. 2). However, their urinary AQP2 levels were 1,00010,000 -fold less than that of their healthy relative. In urine of the healthy individual, glycosylated AQP2 (40-45 kD) was detected also. During dDAVP infusion studies both patients transiently increased their urinary osmolalities to $\sim 350 \mathrm{mmol} / \mathrm{kg}$ $\mathrm{H}_{2} \mathrm{O}$ (Fig. 3). Urine osmolalities of patients with central diabetes insipidus, who lack vasopressin, increased to $600 \mathrm{mmol} / \mathrm{kg}$ $\mathrm{H}_{2} \mathrm{O}$ and remained elevated for the time measured. In patients with X-linked NDI ( $V 2 R$ mutations) or patients with autosomal recessive NDI ( $A Q P 2$ mutations), the urine osmolality

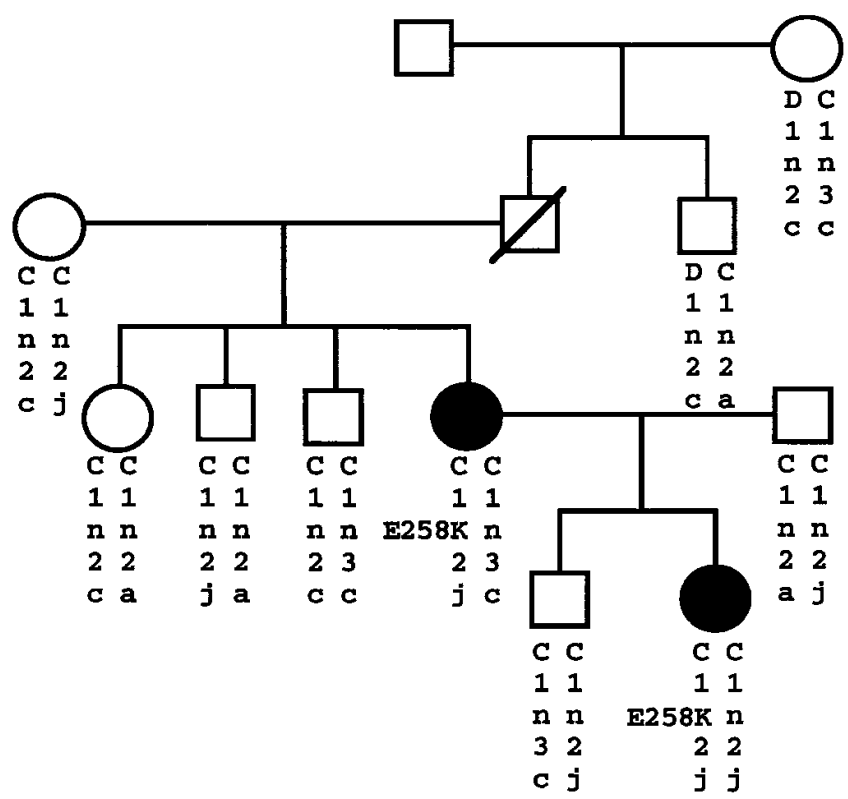

Figure 1. Pedigree with an autosomal dominant inheritance of NDI. The two solid symbols indicate the two affected patients heterozygous for the E258K mutation. The $12 \mathrm{q} 13$ haplotype is represented and the marker order: centromere-Col2A1-AFM259vf9-AQP2D12S131-AFMb007yg5-telomere has been determined in Dr. Kucherlapati's laboratory (20).

was and stayed below $150 \mathrm{mmol} / \mathrm{kg} \mathrm{H}_{2} \mathrm{O}$ (Fig. 3). All the members of the family presented here were examined and tested but no other family members besides the proband and her daughter demonstrated polyuric-polydipsic symptoms.

Phosphorylation of AQP2-E258K is not altered in dominant NDI. To investigate the molecular cause of NDI in these patients, Xenopus oocytes were used as a model system. The E258K mutation is located two residues downstream from serine 256, the residue which is phosphorylated upon stimulation of collecting duct cells by AVP (27). In an LLC-PK 1 cell model, it has been shown that phosphorylation of this serine is

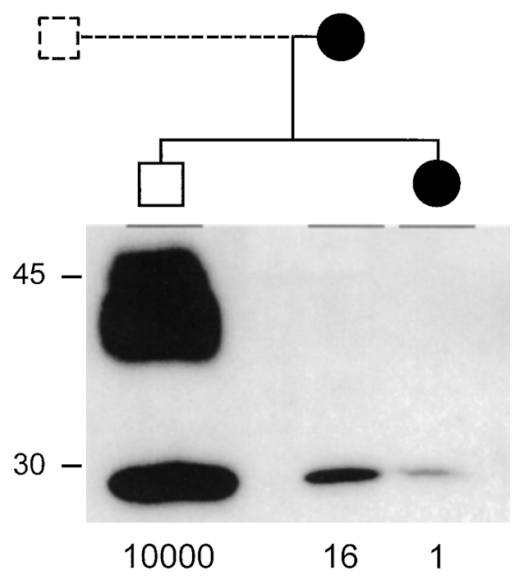

Figure 2. Urinary AQP2 expression in members of the family with dominant inheritance of NDI. Morning urinary samples from the proband, affected daughter, and healthy son were concentrated and creatinine equivalents were immunoblotted for AQP2. Shown is part of the pedigree as shown in Fig. 1. The affected patients are indicated by solid symbols. The relative amounts

of AQP2, as determined by densitometry of the obtained signals, are indicated below particular lanes. The molecular masses of marker proteins are indicated on the left. 


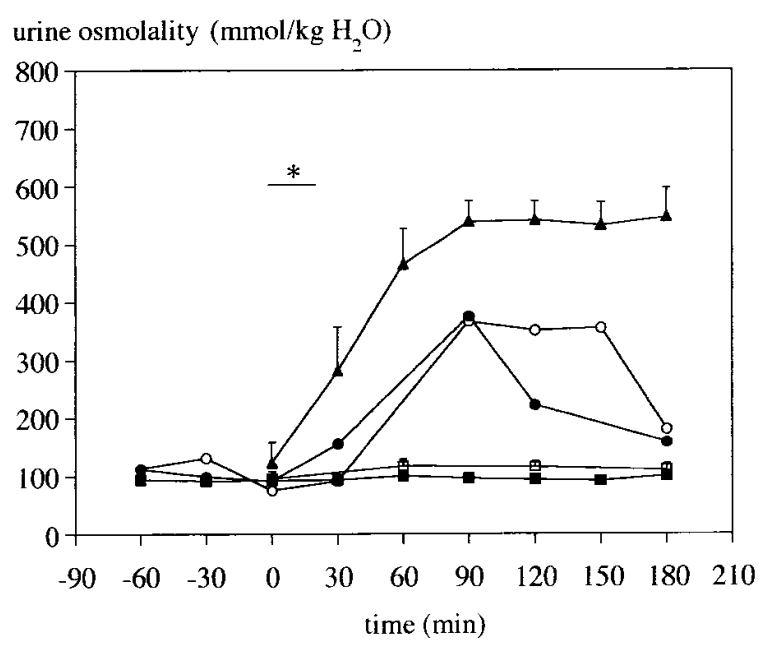

Figure 3. Urinary osmolality of affected individuals in dominant NDI. Urinary osmolality (mean $\pm \mathrm{SE}$ ) was measured before and after dDAVP infusion (*dDAVP was infused from 0 to $20 \mathrm{~min}$ ). Shown are the urine osmolalities of the proband (open circles), her daughter (filled circles), nine patients with V2R mutations (open boxes), three patients homozygous for $A Q P 2$ mutations (filled boxes), and four patients with central diabetes insipidus (filled triangles).

critical for vesicles containing AQP2 to fuse with the plasma membrane upon activation by forskolin $(28,29)$. To find out whether altered phosphorylation of S256 in the AQP2-E258K mutant causes NDI in these patients, AQP2-E258K and AQP2-S256A, in which the PKA phosphorylation site S256 was changed into an alanine, were analyzed in Xenopus oocytes (Fig. 4). Phosphorylation experiments were performed with oocytes injected with $10 \mathrm{ng}$ cRNA encoding wt-AQP2,

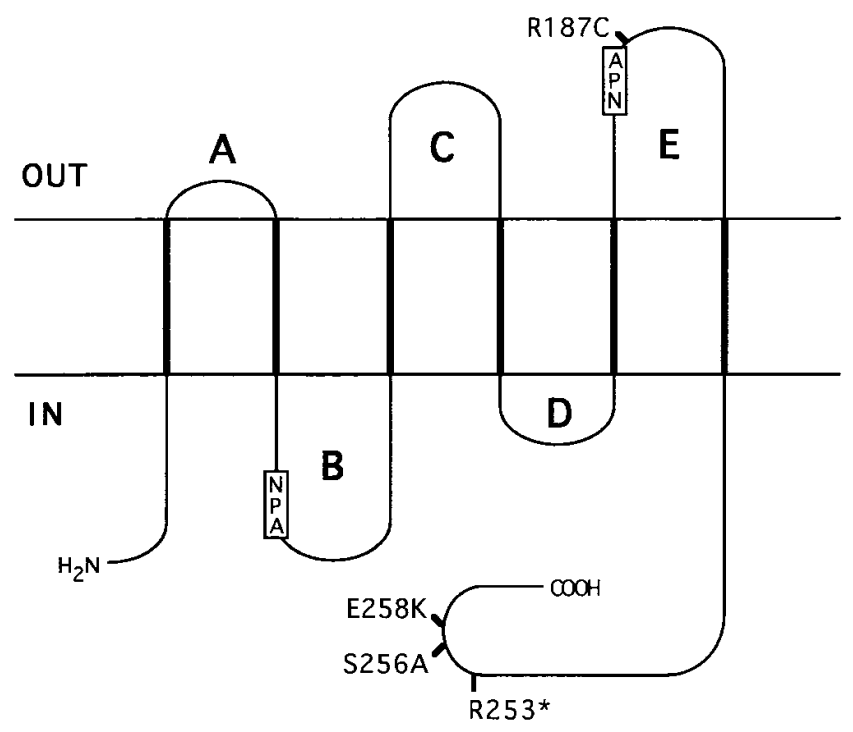

Figure 4. Location of the studied mutations in the topology model of AQP2. Indicated are the amino acid substitutions causing the dominant form of NDI (E258K), the phosphorylation mutant S256A, the truncation mutant R253*, and the mutant R187C found in recessive NDI.

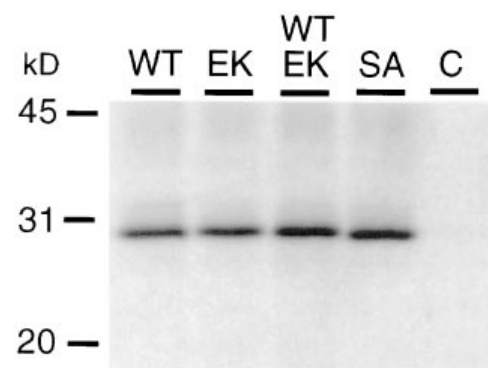

A

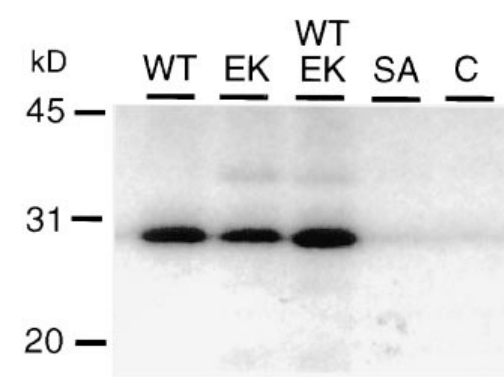

B

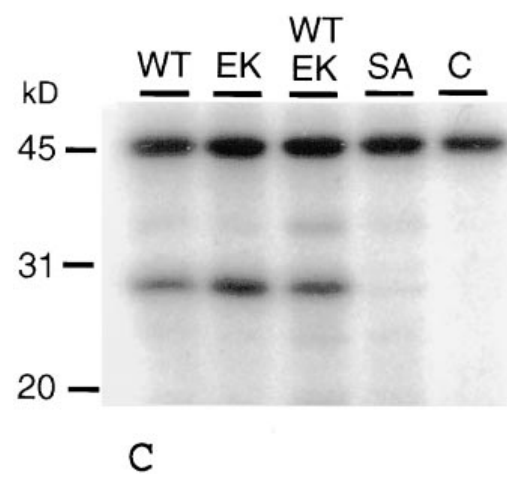

Figure 5. Immunoprecipitation and phosphorylation of AQP2 proteins. Oocytes were injected with water $(C)$ or with $10 \mathrm{ng}$ of cRNA encoding wt-AQP2 (WT), AQP2-E258K $(E K)$, or AQP2-S256A $(S A)$, or 5 ng of both wtAQP2 and AQP2E258K cRNA ( $W T$ ) $E K)$. (A) After injection, newly synthesized proteins were metabolically labeled with ${ }^{35}$ S-labeled methionine for $2 \mathrm{~d}$. Next, oocytes were homogenized and AQP2 proteins were immunoprecipitated with an AQP2 antibody and subjected to SDSPAGE. (B) $2 \mathrm{~d}$ after injection, oocytes were homogenized and proteins were labeled with $\left[\gamma^{-32} \mathrm{P}\right] \mathrm{ATP}$. AQP2 proteins were immunoprecipitated and samples were separated by SDSPAGE. (C) $2 \mathrm{~d}$ after injection, oocytes were homogenized and AQP2 proteins were immunoprecipitated. After immunoprecipitation, AQP2 proteins were phosphorylated using $\left[\gamma^{-32} \mathrm{P}\right] \mathrm{ATP}$ and PKA, and separated by SDS-PAGE.
AQP2-E258K, or AQP2-S256A, and in oocytes injected with both $5 \mathrm{ng}$ wt-AQP2 cRNA and $5 \mathrm{ng}$ AQP2-E258K cRNA (wt + EK). SDS-PAGE of immunoprecipitated AQP2 proteins from these oocytes, labeled with $\left[{ }^{35} \mathrm{~S}\right]$ methionine, revealed similar signals, which indicates that the efficiencies of immunoprecipitation of wt-AQP2, AQP2-E258K, and AQP2-S256A are similar (Fig. $5 \mathrm{~A}$ ). To determine whether the E258K mutation interferes with phosphorylation of S256 and to exclude possible differences in extent of phosphorylation because of putative differences in compartmentalization of the AQP2 proteins in oocytes, AQP2 proteins from oocytes of one batch were phosphorylated directly after homogenization, or after immunoprecipitation. In the oocyte homogenate, phosphorylation of wtAQP2, AQP2-E258K, and AQP2 wt + EK was identical, and as expected, AQP2-S256A was not phosphorylated (Fig. $5 \mathrm{~B}$ ). Phosphorylation of wt-AQP2, AQP2 wt + EK, and AQP2E258K after immunoprecipitation yielded similar results (Fig. $5 \mathrm{C}$ ). The radioactive band around $45 \mathrm{kD}$ was present in all lanes and most likely represents phosphorylated immunoglobulin heavy chains. 


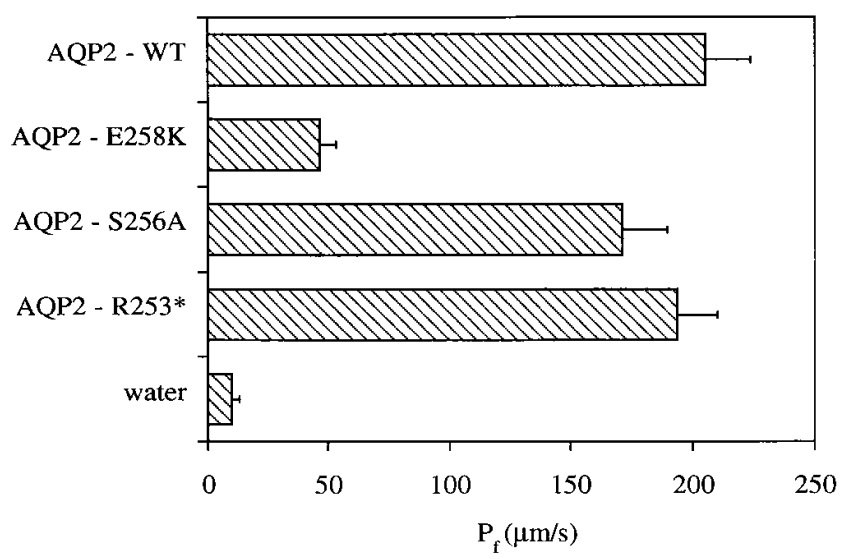

Figure 6. Osmotic $\mathrm{P}_{\mathrm{f}}$ of oocytes expressing wild-type or mutant $\mathrm{AQP} 2$ proteins. $3 \mathrm{~d}$ after injection of water or $10 \mathrm{ng}$ of cRNA encoding wt-AQP2, AQP2-E258K, AQP2-S256A, or AQP2-R253*, $\mathrm{P}_{\mathrm{f}} \mathrm{s}$ were measured in a standard swelling assay. Means \pm SE of at least 40 oocytes from 4 different experiments are shown.

AQP2-E258K confers a dominant-negative effect on the function of $w t-A Q P 2$. We next investigated the $\mathrm{P}_{\mathrm{f}}$ of oocytes microinjected with various AQP2 cRNAs. $3 \mathrm{~d}$ after injection of 10 ng of cRNA, $\mathrm{P}_{\mathrm{f}} \mathrm{S}\left(\mathrm{P}_{\mathrm{f}} \pm \mathrm{SE}\right)$ of oocytes expressing wt-AQP2, AQP2-S256A, or AQP2-R253*, which lacks the COOH terminus of AQP2 following arginine 253 except for the last two amino acids, were not significantly different (Fig. 6). However, the $\mathrm{P}_{\mathrm{f}}$ of oocytes expressing the E258K mutant was low, but still significantly higher than of control oocytes (Fig. 6). To investigate whether AQP2-E258K conferred a dominant-negative effect on the function of wt-AQP2, oocytes were injected with $0.3 \mathrm{ng}$ wt-AQP2 alone or together with $0.3 \mathrm{ng}$ AQP2E258K cRNA. As a control, oocytes were also injected with 0.3 ng wt-AQP2 cRNA together with 0.3 ng cRNA encoding AQP2-R187C, which is a stable AQP2 mutant found in recessive NDI $(7,15) .2 \mathrm{~d}$ after injection, the $\mathrm{P}_{\mathrm{f}}$ of oocytes expressing wt-AQP2/AQP2-E258K was significantly lower than the $P_{f}$ of oocytes expressing wt-AQP2/AQP2-R187C or wt-AQP2 alone and was not significantly different from water-injected oocytes or oocytes injected with just $0.3 \mathrm{ng}$ AQP2-E258K cRNA (Fig. $7 A$ ). The $\mathrm{P}_{\mathrm{f}}$ of oocytes injected with $0.3 \mathrm{ng}$ AQP2R187C cRNA was not different from controls, which shows that this mutant did not contribute to the $P_{f}$ of the oocytes in which this mutant was coexpressed with wt-AQP2. To check whether the observed dominant effect was not caused by a higher expression level of AQP2-E258K compared with AQP2-R187C, lysates of these oocytes were immunoblotted (Fig. $7 \mathrm{~B}$ ). Densitometric scanning of the obtained signals revealed that oocytes injected with $0.3 \mathrm{ng}$ of AQP2-R187C cRNA alone or in combination with wt-AQP2 cRNA expressed a higher amount of AQP2 than the oocytes injected with AQP2-E258K cRNA alone or in combination with wtAQP2, respectively. These results clearly show that the AQP2 mutant in dominant NDI (AQP2-E258K), but not the mutant in recessive NDI (AQP2-R187C), has a dominant-negative effect on the function of wt-AQP2 in oocytes.

Altered intracellular transport of AQP2-E258K causes dominant NDI. Because we observed previously that the autosomal recessive form of NDI is caused by mutations in AQP2 that result in retention of mutant proteins in the ER $(15,16)$, we next addressed the intracellular distribution of the AQP2-E258K mutant. Immunoblotting of lysates from oocytes injected with $10 \mathrm{ng}$ of cRNA encoding wt-AQP2, AQP2E258K, AQP2-S256A, AQP2-R253*, or 5 ng of both wt-AQP2 cRNA and AQP2-E258K cRNA, revealed that equal amounts of protein were expressed (Fig. $8 A$ ). The wt and missense AQP2 proteins run at a size of $29 \mathrm{kD}$. The AQP2-R253* is $\sim 2 \mathrm{kD}$ smaller because this protein lacks 16 amino acids of the $\mathrm{COOH}$-terminal tail. No signal was obtained in the lane loaded with lysates from water-injected control oocytes. To determine the plasma membrane expression of wild-type and mutant AQP2 proteins, fractions enriched for plasma membranes were isolated from oocytes and subjected to immunoblotting (Fig. 8 B). AQP2-S256A, AQP2-R253*, and wt-AQP2 expressing oocytes showed a comparable level of expression at the plasma membrane. In contrast, the expression of the E258K mutant on the plasma membrane was much lower.

Intracellular localization of $w t-A Q P 2$ and $A Q P 2-E 258 K$
A

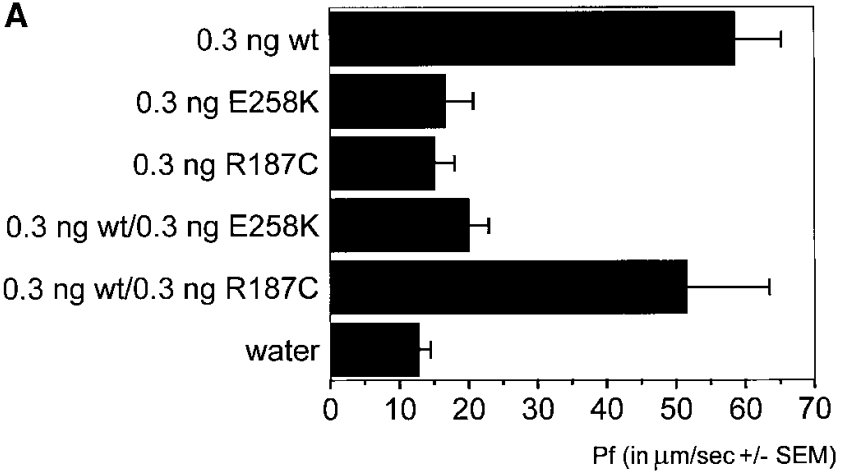

B

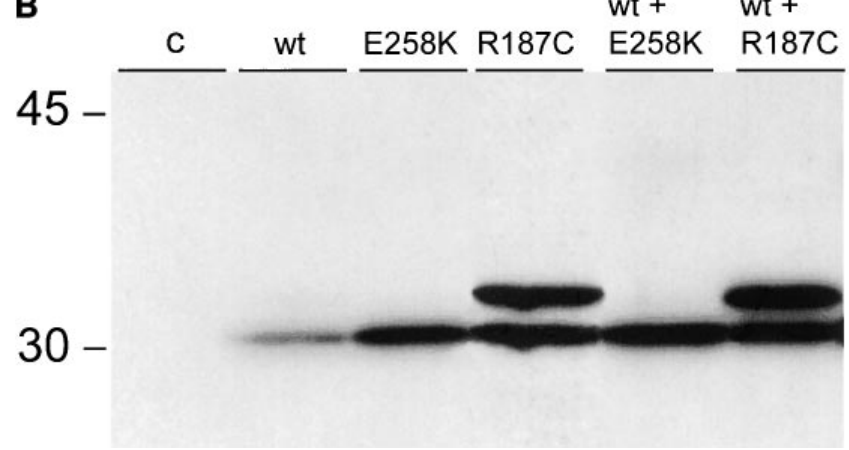

Figure 7. Osmotic $\mathrm{P}_{\mathrm{f}}$ of oocytes expressing wild-type and mutant AQP2 proteins. $(A) 2 \mathrm{~d}$ after injection of water (water) or $0.3 \mathrm{ng}$ of cRNA encoding wt-AQP2 (wt), AQP2-E258K (E258K), or AQP2-R187C (R187C) alone or 0.3 ng cRNA encoding wt-AQP2 in combination with 0.3 ng AQP2-E258K $(w t / E 258 K)$ or AQP2-R187C $(w t / R 187 C)$ cRNAs, $\mathrm{P}_{\mathrm{f}} \mathrm{s}$ were measured in a standard swelling assay. Shown are the mean \pm SE of at least 10 oocytes. $(B)$ Oocytes of which the $\mathrm{P}_{\mathrm{f}}$ was determined as shown in $A$ were homogenized and immunoblotted. After incubation of the membrane with affinity-purified rabbit AQP2 antibodies, followed by affinity-purified goat anti-rabbit IgG coupled to horseradish peroxidase, AQP2 proteins were visualized using enhanced chemiluminescence. 


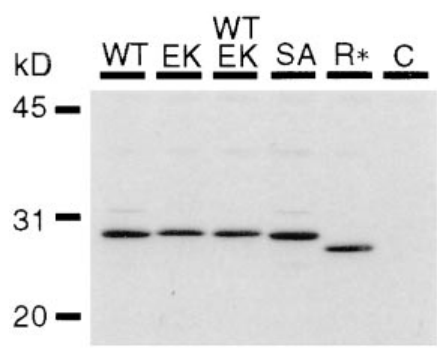

A

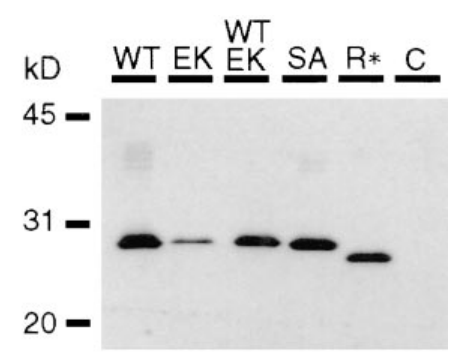

B
Figure 8. Immunoblot analysis of wild-type and mutant AQP2 proteins expressed in oocytes. $(A)$ $3 \mathrm{~d}$ after injection, homogenates were prepared from eight oocytes injected with water $(C)$ or $10 \mathrm{ng}$ of cRNA encoding wt, E258K, wt + E258K (5 ng each), S256A, or R253* AQP2. Equivalents of 0.1 oocyte were separated by SDS-PAGE and immunoblotted. (B) Plasma membranes were prepared $3 \mathrm{~d}$ after injection of oocytes injected with water or cRNA encoding wt, E258K, wt + E258K (5 ng each), S256A, or R253* AQP2. Equivalents of eight oocytes were separated by SDS-PAGE and AQP2 expression was analyzed by immunoblotting as described in the legend to Fig. $7 \mathrm{~B}$. protein. To precisely define the intracellular localization of the AQP2-E258K protein, we performed immunofluorescence microscopy on sections prepared from paraformaldehydefixed oocytes (Fig. 9). Oocytes expressing wt-AQP2 showed a discrete, intense staining of the plasma membrane. In contrast, in oocytes expressing the AQP2-E258K mutant protein only a very faint labeling of the plasma membrane was observed. Most of AQP2-E258K mutant protein was confined to a cytoplasmic region just underneath the plasma membrane. Oocytes expressing AQP2-S256A or AQP2-R253* show a distinct staining of the plasma membrane, but it is clear that part of these mutant proteins is also expressed just below the plasma membrane. Noninjected control oocytes showed no staining with the AQP2 antibody. In contrast, oocytes expressing AQP2-T126M, which is a mutant in recessive NDI, reveal a disperse staining (Fig. 9 E) (16). At the ultrastructural level, we found that wt-AQP2 was primarily localized to the plasma membrane and the Golgi apparatus. In contrast, in oocytes expressing AQP2-E258K, labeling was localized to the Golgi apparatus and intracellular vesicles, whereas staining of the plasma membrane was essentially negligible (Fig. 10). Control oocytes were again negative for AQP2.

\section{Discussion}

Recently, we have shown that mutations in the $A Q P 2$ gene cause the autosomal recessive form of NDI $(7,8,16)$. Here, we report for the first time that the autosomal dominant form of inheritance of NDI is also caused by a mutation in the AQP2 gene. Sequence analysis of the $A Q P 2$ genes of the family of the patient showed that the affected patient and her daughter carried one mutant allele, encoding a E258K substitution, whereas nonaffected family members were homozygous for the wild-type allele (Fig. 1). In line with this, the urinary AQP2 level in the affected individuals was $>1,000$-fold less than in their healthy relative (Fig. 2). Previous analysis of urine of patients with recessive NDI caused by mutations in the AQP2 gene revealed no detectable AQP2, which was explained by a low stability of these AQP2 mutants $(30,31)$. The detection of low AQP2 levels in urine of the NDI patients in this study is in agreement with the ability of these patients to transiently increase their urine after dDAVP infusion (Fig. 3). Expressed in Xenopus oocytes, AQP2-E258K was as stable as wt-AQP2. However, since it is unknown whether the detected urinary AQP2 consist of wt-AQP2 and AQP2-E258K or wt-AQP2 only, this approach cannot address the question of whether AQP2-E258K is stable in vivo.
Interestingly, all AQP2 mutations reported so far in recessive NDI were located between the first and last transmembrane domain $(7,8,16)$, whereas the new mutation in dominant NDI is located in the COOH-terminal tail. This suggests a relationship between the site of mutation and the inheritance pattern. As shown for other diseases, a dominant form of inheritance occurs when a mutant protein oligomerizes with other subunits of a functional complex and disturbs the routing or the function of the complex. Aquaporins are known to oligomerize and are present in the plasma membrane as homotetramers $(32,33)$, but it has been shown that the monomer is the functional unit (34). Therefore, the dominant action of AQP2 mutants in NDI can only be explained if the mutant is able to oligomerize with wt-AQP2 and the complex is perturbed in its routing after oligomerization. It remains to be established in which organelle oligomerization of AQP2 occurs. Likely candidates include the ER and trans-Golgi network that are known as the assembly sites of multiprotein complexes like the $\mathrm{T}$ cell antigen receptor and the gap junction protein connexin-43 (35-37).

Since the E258K mutation is located close to the PKA phosphorylation site S256, we hypothesized that this mutation might interfere with phosphorylation of S256. However, from Fig. 5 it is clear that the E258K mutation did not interfere with phosphorylation of S256 and therefore this explanation for dominant NDI can be excluded. To further investigate the molecular cause of NDI in these patients we compared the function and routing of AQP2-E258K with wt-AQP2, AQP2S256A, and AQP2-R253*. Swelling tests of oocytes expressing AQP2-E258K revealed a low $\mathrm{P}_{\mathrm{f}}$, whereas the $\mathrm{P}_{\mathrm{f}} \mathrm{s}$ of AQP2S256A, AQP2-R253*, and wt-AQP2 were significantly higher (Fig. 6). These obtained $\mathrm{P}_{\mathrm{f}} \mathrm{s}$ were not a consequence of differences in expression levels, since these were similar (Fig. $8 \mathrm{~A}$ ), but were directly related to the expression levels at the plasma membrane (Fig. $8 \mathrm{~B}$ and 9). Sections of oocytes expressing AQP2-S256A or AQP2-R253* showed, in addition to a considerable amount of expression at the plasma membrane, some protein in the region just below the plasma membrane (Fig. 9). This suggests that phosphorylation is involved in AQP2 transport to the plasma membrane in oocytes. Such effects on trafficking in response to phosphorylation have also been shown for the $\mathrm{Na}^{+}$/glucose cotransporter expressed in oocytes (38), and might explain the increase in AQP2-mediated water transport in oocytes after treatment with cAMP (27). The obtained $\mathrm{P}_{\mathrm{f}}$ and plasma membrane expression of AQP2-S256A and AQP2-R253* contrasts with results obtained in LLC-PK 1 cells which were stably transfected with the AQP2-S256A $(28,29)$. 

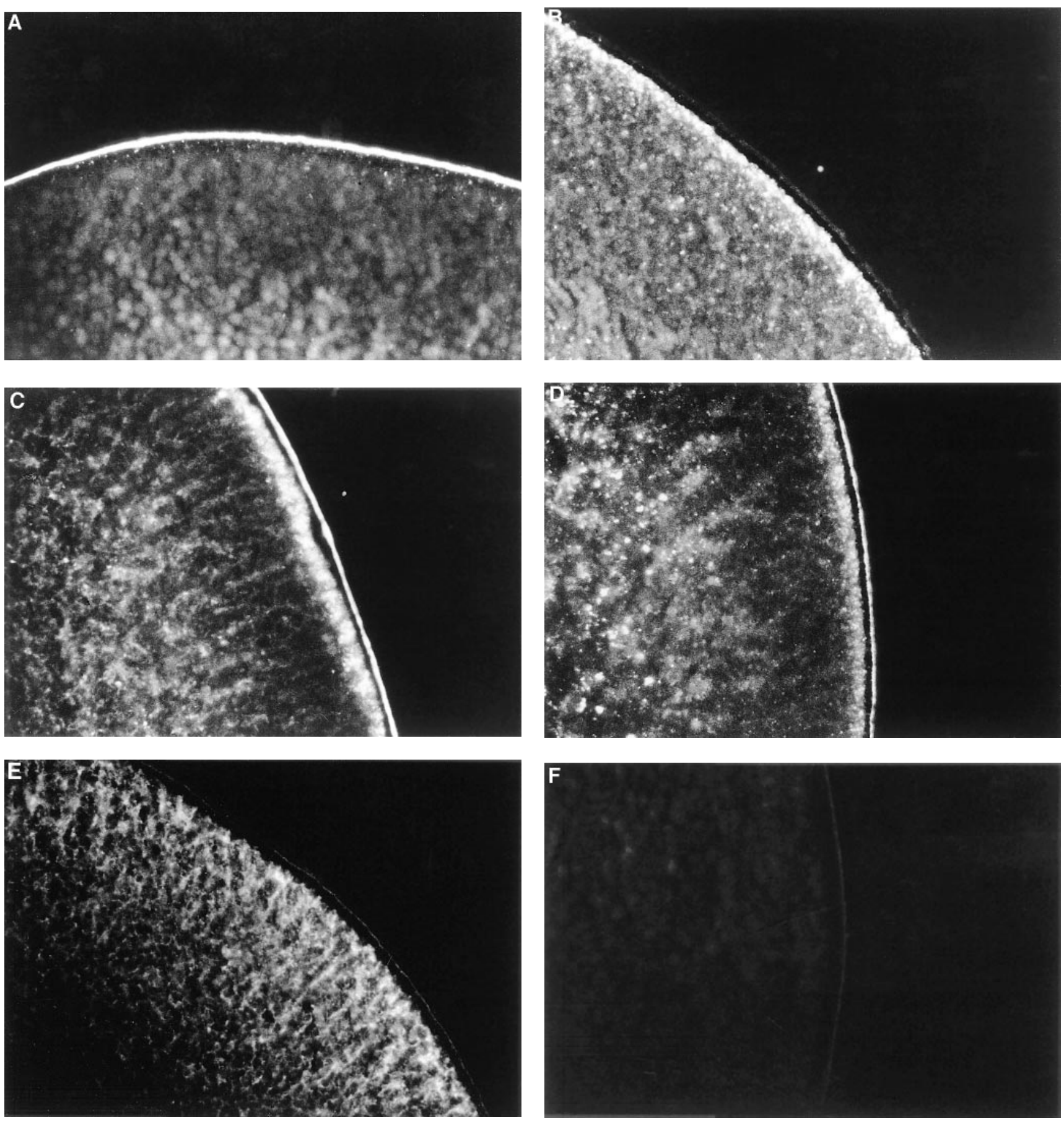

Figure 9. Immunofluorescence microscopy of wild-type and mutant AQP2 proteins in oocytes. $3 \mathrm{~d}$ after injection with cRNAs encoding wt $(A)$, AQP2-E258K $(B)$, AQP2-S256A $(C)$, AQP2-R253* $(D)$, or AQP2-T126M (E), oocytes were fixed in paraformaldehyde. As a negative control, water-injected oocytes were used $(F)$. To visualize AQP2, the sections were incubated with affinity-purified rabbit AQP2 antibodies as primary immunoglobulins and FITC-conjugated anti-rabbit IgGs as secondary antibodies.

This discrepancy is likely to be a consequence of relatively higher expression levels of the AQP mutants in oocytes, saturating the "shuttling" machinery in oocytes. A similar phenomenon has been found for the most prevalent mutant in the cystic fibrosis transmembrane conductance regulator, CFTR $\Delta$ F508. In oocytes, this common CFTR mutant is retarded in its export from the ER, but a small fraction is found at the plasma membrane (39), whereas this mutant was not found at the plasma membrane of mammalian cells (40). The low plasma membrane expression levels of AQP2-E258K in comparison to AQP2-S256A and AQP2-R253* underscore the routing impairment of AQP2-E258K.

The appearance of AQP2-E258K on immunoblot is remarkably different from AQP2 mutants in recessive NDI. The 

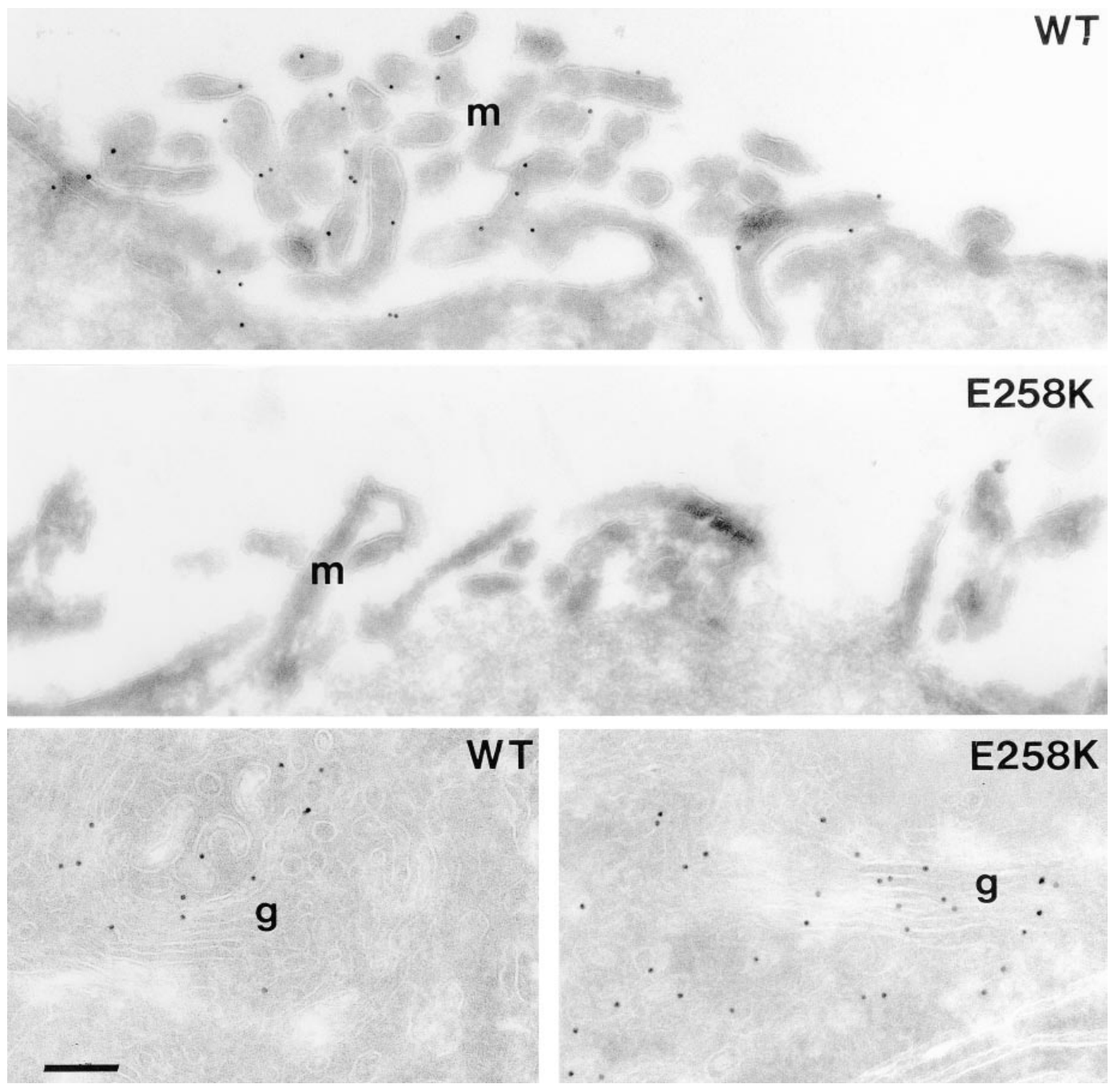

Figure 10. Immunogold electronmicroscopy of expressed AQP2 proteins in oocytes. Ultrathin cryosections, prepared from oocytes expressing wtAQP2 or AQP2-E258K, were incubated with affinity-purified AQP2 antibody followed by 10-nm-sized protein A-gold. In oocytes expressing wt-AQP2, most of the gold labeling was observed on microvilli $(m)$ of the plasma membrane, while tubulovesicular structures in the Golgi region, including the Golgi apparatus, were also labeled $(g)$. In oocytes expressing AQP2-E258K, however, only the Golgi region was labeled, whereas no labeling of the plasma membrane was found. Bar denotes $200 \mathrm{~nm}$. latter mutants have a very characteristic additional band of $\sim 32 \mathrm{kD}$ on immunoblots, which was shown previously to be an ER-retarded form of AQP2 $(15,16)$. Immunofluorescence microscopy revealed that the majority of AQP2-E258K was present just underneath the plasma membrane, which differs significantly from ER-retarded mutant AQP2 proteins, which are evenly distributed in the cytoplasm (Fig. 9) $(15,16)$. Immunogold electronmicroscopy further showed that AQP2-E258K was localized exclusively in the Golgi area, while wt-AQP2 was predominantly associated with the plasma membrane and some labeling of the Golgi complex and related tubulovesicular structures (Fig. 10). The pool of wt-AQP2 molecules in the Golgi apparatus probably reflects newly synthesized protein en route to the plasma membrane, but the localization of AQP2-E258K in the Golgi apparatus indicates that this mutant is retained in this organelle. In coexpression experiments, expression of low levels of AQP2-E258K, but not AQP2$\mathrm{R} 187 \mathrm{C}$, appeared to interfere with the function of wt-AQP2 (Fig. 7). The lower expression level of AQP2-E258K compared with that of AQP2-R187C excludes the possibility that a saturation of the translation machinery leading to a reduced expression level of wt-AQP2 causes the dominant effect of AQP2-E258K. Since AQPs form tetramers $(32,33)$ and
AQP2-E258K is piled up in the Golgi apparatus (Fig. 10), the observed dominant effect is presumably caused by an impaired routing of wt-AQP2/AQP2-E258K tetramers to the plasma membrane. In oocytes, proper oligomerization of subunits has been found for the dimeric $\mathrm{Na} / \mathrm{K}$-ATPase, and the tetrameric sodium channel, which also occur in functional complexes at the plasma membrane $(41,42)$. Missense mutations leading to mistargeting have also been found in other diseases. In primary hyperoxaluria, a mutation in the alanine-glyoxalate aminotransferase results in the targeting of this enzyme to mitochondria instead of peroxisomes (43). Also, in Liddle syndrome a mutation in the tail of the $\alpha$ or $\beta$ subunit of the sodium channel destroys the coated pit internalization signal, resulting in a constitutive expression of the sodium channel at the plasma membrane, increased $\mathrm{Na}^{+}$absorption, and hypertension (42).

Since the $\mathrm{P}_{\mathrm{f}} \mathrm{s}$ of oocytes expressing wt-AQP2, AQP2S256A, or AQP2-R253* were not different, the 16 amino acids of the $\mathrm{COOH}$-terminal tail, including the region of the E258K mutation, and phosphorylation of S256 in particular are, at these expression levels, not essential for targeting of AQP2 to the plasma membrane of oocytes. Therefore, it is very likely that the change from a negative into a positive charge in the 
E258K mutant introduces a Golgi retention signal or a Golgidirected internalization signal instead of removing an intrinsic AQP2 routing signal. The transient increase in urine osmolalities in our patients with dominant NDI in response to dDAVP, compared with the sustained increase in urine osmolalities of patients with central diabetes insipidus (Fig. 3), suggests that the tetrameric aquaporin complexes are internalized faster from the plasma membrane and is in favor of an introduced endocytotic signal in AQP2-E258K. Interestingly, in the transGolgi network protein of $38 \mathrm{kD}$ (TGN38), a YQRL motif has been found to direct its endocytosis and routing to the transGolgi compartment (44). However, since the tyrosine, which is absent in AQP2-E258K, was found to be essential, the mechanisms of Golgi retainment of AQP2-E258K and TGN38 are likely to differ.

In conclusion, we report here that autosomal dominant NDI is caused by a mutation in the $A Q P 2$ gene. In contrast to AQP2 mutants in recessive NDI, which are all impaired in their export from the ER, the E258K mutant was retained in the Golgi apparatus, presumably because the substitution introduces a signal for Golgi retention. In mixed tetramers of wt-AQP2 and AQP2-E258K, intracellular retention of the AQP2-E258K mutant in the Golgi offers an explanation for the dominant form of inheritance of NDI in the described family.

\section{Acknowledgments}

We thank Dr. R.J.M. Bindels for valuable suggestions.

This study was supported by grants from the Life Sciences Foundation (NWO-SLW-810-405-16.2) to S.M. Mulders, the Dutch Kidney Foundation (C95.5001) to J.P.L. Rijss, E.J. Kamsteeg, and P.M.T. Deen, the Dutch Diabetes Foundation (94.110), and the Ludgardine Bouwman-Jan Dekker Foundation to P. van der Sluijs. P.M.T. Deen and P. van der Sluijs are investigators of the Royal Netherlands Academy of Arts and Sciences.

\section{References}

1. Preston, G.M., T.P. Carroll, W.B. Guggino, and P. Agre. 1992. Appearance of water channels in Xenopus oocytes expressing red cell CHIP28 protein. Science. 256:385-387.

2. Fushimi, K., S. Uchida, Y. Hara, Y. Hirata, F. Marumo, and S. Sasaki. 1993. Cloning and expression of apical membrane water channel of rat kidney collecting tubule. Nature. 361:549-552.

3. Echevarria, M., E.E. Windhager, S.S. Tate, and G. Frindt. 1994. Cloning and expression of AQP3, a water channel from the medullary collecting duct of rat kidney. Proc. Natl. Acad. Sci. USA. 91:10997-11001.

4. Ishibashi, K., S. Sasaki, K. Fushimi, S. Uchida, M. Kuwahara, H. Saito, T. Furukawa, K. Nakajima, Y. Yamaguchi, T. Gojobori, et al. 1994. Molecular cloning and expression of a member of the aquaporin family with permeability to glycerol and urea in addition to water expressed at the basolateral membrane of kidney collecting duct cells. Proc. Natl. Acad. Sci. USA. 91:6269-6273.

5. Ma, T., A. Frigeri, H. Hasegawa, and A.S. Verkman. 1994. Cloning of a water channel homolog expressed in brain meningeal cells and kidney collecting duct that functions as a stilbene-sensitive glycerol transporter. J. Biol. Chem. 269:21845-21849.

6. Jung, J.S., R.V. Bhat, G.M. Preston, W.B. Guggino, J.M. Baraban, and P. Agre. 1994. Molecular characterization of an aquaporin cDNA from brain: candidate osmoreceptor and regulator of water balance. Proc. Natl. Acad. Sci. USA. 91:13052-13056.

7. Deen, P.M.T., M.A. Verdijk, N.V.A.M. Knoers, B. Wieringa, L.A.H. Monnens, C.H. van Os, and B.A. van Oost. 1994. Requirement of human renal water channel aquaporin-2 for vasopressin-dependent concentration of urine. Science. 264:92-95.

8. Van Lieburg, A.F., M.A. Verdijk, N.V.A.M. Knoers, A.J. van Essen, W. Proesmans, R. Mallmann, L.A.H. Monnens, B.A. van Oost, C.H. van Os, and P.M.T. Deen. 1994. Patients with autosomal nephrogenic diabetes insipidus homozygous for mutations in the aquaporin 2 water-channel gene. Am. J. Hum.
Genet. 55:648-652.

9. Marples, D., M.A. Knepper, E.I. Christensen, and S. Nielsen. 1995. Redistribution of aquaporin-2 water channels induced by vasopressin in rat kidney inner medullary collecting duct. Am. J. Physiol. 38:C655-C664.

10. Nielsen, S., C.L. Chou, D. Marples, E.I. Christensen, B.K. Kishore, and M.A. Knepper. 1995. Vasopressin increases water permeability of kidney collecting duct by inducing translocation of aquaporin-CD water channels to plasma membrane. Proc. Natl. Acad. Sci. USA. 92:1013-1017.

11. Katsura, T., J.M. Verbavatz, J. Farinas, T. Ma, D.A. Ausiello, A.S Verkman, and D. Brown. 1995. Constitutive and regulated membrane expression of aquaporin 1 and aquaporin 2 water channels in stably transfected LLCPK1 epithelial cells. Proc. Natl. Acad. Sci. USA. 92:7212-7216.

12. Yamamoto, T., S. Sasaki, K. Fushimi, K. Ishibashi, E. Yaoita, K. Kawasaki, F. Marumo, and I. Kihara. 1995. Vasopressin increases AQP-CD water channel in apical membrane of collecting duct cells in Brattleboro rats. Am. J. Physiol. 268:C1546-C1551.

13. Van den Ouweland, A.M., J.C. Dreesen, M.A. Verdijk, and N.V.A.M. Knoers. 1992. Mutations in the vasopressin type 2 receptor gene (AVPR2) associated with nephrogenic diabetes insipidus. Nat. Genet. 2:99-102.

14. Rosenthal, W., A. Seibold, A. Antaramian, M. Lonergan, M.-F. Arthus, G.N. Hendy, M. Birnbaumer, and D.G. Bichet. 1992. Molecular identification of the gene responsible for congenital nephrogenic diabetes insipidus. Nature. 359:233-235.

15. Deen, P.M.T., H. Croes, R.A. van Aubel, L.A. Ginsel, and C.H. van Os. 1995. Water channels encoded by mutant aquaporin-2 genes in nephrogenic diabetes insipidus are impaired in their cellular routing. J. Clin. Invest. 95:22912296.

16. Mulders, S.M., N.V.A.M. Knoers, A.F. van Lieburg, L.A.H. Monnens, E. Leumann, E. Wuhl, E. Schober, J.P.L. Rijss, C.H. van Os, and P.M.T. Deen 1997. New mutations in the AQP2 gene in nephrogenic diabetes insipidus resulting in functional but misrouted water channels. J. Am. Soc. Nephrol. 8:242-248.

17. Bichet, D.G., M. Razi, M. Lonergan, M.-F. Arthus, V. Papukna, C. Kortas, and J.N. Barjon. 1988. Hemodynamic and coagulation responses to 1-desamino [8-D-arginine] vasopressin in patients with congenital nephrogenic diabetes insipidus. N. Engl. J. Med. 318:881-887.

18. Bichet, D.G., M.-F. Arthus, M. Lonergan, G.N. Hendy, A.J. Paradis, T.M. Fujiwara, K. Morgan, M.C. Gregory, W. Rosenthal, A. Didwania, et al 1993. X-linked nephrogenic diabetes insipidus mutations in North America and the Hopewell hypothesis. J. Clin. Invest. 92:1262-1268.

19. Bichet, D.G., M. Birnbaumer, M. Lonergan, M.-F. Arthus, and W. Rosenthal. 1994. Nature and recurrence of AVPR2 mutations in X-linked nephrogenic. Am. J. Hum. Genet. 55:278-286.

20. LeBlanc-Straceski, J.M., K.T. Montgomery, H. Kissel, L. Murthaugh, P. Tsai, D.C. Ward, K.S. Krauter, and R. Kucherlapati. 1994. Twenty-one polymorphic markers from human chromosome 12 for integration of genetic and physical maps. Genomics. 19:341-349.

21. Hattori, M., and Y. Sakaki. 1986. Dideoxy sequencing method using denatured plasmid templates. Anal. Biochem. 152:232-238.

22. Wall, D.A., and S. Patel. 1989. Isolation of plasma membrane complexes from Xenopus oocytes. J. Membr. Biol. 107:189-201.

23. Laemmli, U.K. 1970. Cleavage of structural proteins during the assembly of the head of bacteriophage $\mathrm{T}_{4}$. Nature. 227:680-685.

24. McLean, I.W., and P.K. Nakane. 1974. Periodate-lysine-paraformaldehyde fixative. A new fixation for immunoelectron microscopy. J. Histochem. Cytochem. 22:1077-1083.

25. Hansen, S.H., K. Sandvig, and B. van Deurs. 1993. Molecules internalized by clathrin-independent endocytosis are delivered to endosomes containing transferrin receptors. J. Cell Biol. 123:89-97.

26. James, D.E., R.C. Piper, and J.W. Slot. 1993. Targeting of mammalian glucose transporters. J. Cell Sci. 104:607-612.

27. Kuwahara, M., K. Fushimi, Y. Terada, L. Bai, F. Marumo, and S. Sasaki. 1995. cAMP-dependent phosphorylation stimulates water permeability of aquaporin-collecting duct water channel protein expressed in Xenopus oocytes. J. Biol. Chem. 270:10384-10387.

28. Katsura, T., C.E. Gustafson, D.A. Ausiello, and D. Brown. 1997. Protein kinase A phosphorylation is involved in regulated exocytosis of aquaporin-2 in transfected LLC-PK1 cells. Am. J. Physiol. 41:F816-F822.

29. Fushimi, K., S. Sasaki, and F. Marumo. 1997. Phosphorylation of serine 256 is required for cAMP-dependent regulatory exocytosis of the aquaporin-2 water channel. J. Biol. Chem. 272:14800-14804.

30. Deen, P.M.T, R.A. van Aubel, A.F van Lieburg, and C.H. van Os. 1996. Urinary content of aquaporin 1 and 2 in nephrogenic diabetes insipidus. $J$. Am. Soc. Nephrol. 7:836-841.

31. Kanno, K., S. Sasaki, Y Hirata, S. Ishikawa, K. Fushimi, S. Nakanishi, D.G. Bichet, and F. Marumo. 1995. Urinary excretion of aquaporin-2 in patients with diabetes insipidus. N. Engl. J. Med. 332:1540-1545.

32. Smith, B.L,. and P. Agre. 1991. Erythrocyte $M_{\mathrm{r}} 28,000$ transmembrane protein exists as a multisubunit oligomer similar to channel proteins. J. Biol. Chem. 266:6407-6415.

33. Verbavatz, J.M., D. Brown, I. Sabolic, G. Valenti, D.A. Ausiello, A.N. Van Hoek, T. Ma, and A.S. Verkman. 1993. Tetrameric assembly of CHIP28 water channels in liposomes and cell membranes: a freeze-fracture study. J. Cell 
Biol. 123:605-618.

34. Van Hoek, A.N., M.L. Hom, L.H. Luthjens, M.D. de Jong, J.A. Dempster, and C.H. van Os. 1991. Functional unit of $30 \mathrm{kD}$ for proximal tubule water channels as revealed by radiation inactivation. J. Biol. Chem. 266:16633-16635.

35. Copeland, C.S., K.P. Zimmer, K.R. Wagner, G.A. Healey, I. Mellman, and A. Helenius. 1988. Folding, trimerization, and transport are sequential events in the biogenesis of influenza virus hemagglutinin. Cell. 53:197-209.

36. De Silva, A., I. Braakman, and A. Helenius. 1993. Posttranslational folding of vesicular stomatitis virus $\mathrm{G}$ protein in the ER: involvement of noncovalent and covalent complexes. J. Cell Biol. 120:647-655.

37. Musil, L.S., and D.A. Goodenough. 1993. Multisubunit assembly of an integral plasma membrane channel protein, gap junction connexin43, occurs after exit from the ER. Cell. 74:1065-1077.

38. Hirsch, J.R., D.D.F. Loo, and E.M. Wright. 1996. Regulation of $\mathrm{Na}^{+} /$ glucose cotransporter expression by protein kinases in Xenopus laevis oocytes. J. Biol. Chem. 271:14740-14746.

39. Drumm, M.L., D.J. Wilkinson, L.S. Smit, R.T. Worrell, T.V. Strong, R.A. Frizzell, D.C. Dawson, and F.S. Collins. 1991. Chloride conductance expressed by delta F508 and other mutant CFTRs in Xenopus oocytes. Science. 254:1797-1799.
40. Cheng, S.H., R.J. Gregory, J. Marshall, S. Paul, D.W. Souza, and G.A. White. 1990. Defective intracellular transport and processing of CFTR is the molecular basis of most cystic fibrosis. Cell. 63:827-834.

41. Beggah, A., P. Mathews, P. Beguin, and K. Geering. 1996. Degradation and endoplasmic reticulum retention of unassembled alpha- and beta-subunits of Na,K-ATPase correlate with interaction of BiP. J. Biol. Chem. 271:2089520902.

42. Schild, L., Y. Lu, I. Gautschi, E. Schneeberger, R.P. Lifton, and B.C. Rossier. 1996. Identification of a PY motif in the epithelial $\mathrm{Na}$ channel subunits as a target sequence for mutations causing channel activation found in Liddle syndrome. EMBO (Eur. Mol. Biol. Organ.) J. 15:2381-2387.

43. Purdue, P.E., J. Allsop, G. Isaya, L.E. Rosenberg, and C.J. Danpure. 1991. Mistargeting of peroxisomal L-alanine:glyoxylate aminotransferase to mitochondria in primary hyperoxaluria patients depends upon activation of a cryptic mitochondrial targeting sequence by a point mutation. Proc. Natl. Acad. Sci. USA. 88:10900-10904.

44. Humphrey, J.S., P.J. Peters, L.C. Yuan, and J.S. Bonifacino. 1993. Localization of TGN38 to the trans-Golgi network: involvement of a cytoplasmic tyrosine-containing sequence. J. Cell Biol. 120:1123-1135. 